\title{
Papillary muscle septalization for functional tricuspid regurgitation: Proof of concept and preliminary clinical experience
}

\author{
Jean-Paul Couetil, MD, ${ }^{\mathrm{a}}$ Francesco Nappi, MD, ${ }^{\mathrm{a}}$ Cristiano Spadaccio, $\mathrm{MD}, \mathrm{PhD},{ }^{\mathrm{b}, \mathrm{c}}$ and \\ Antonio Fiore, MD, ${ }^{\mathrm{d}}$ Saint-Denis, France; Glasgow and Blackpool, United Kingdom; and Creteil, France
}

\footnotetext{
From the ${ }^{\mathrm{a} D e p a r t m e n t}$ of Cardiac Surgery, Centre Cardiologique du Nord, Saint-Denis, France; ${ }^{\mathrm{b}}$ Institute of Cardiovascular and Medical Sciences, University of Glasgow, Glasgow, United Kingdom; ${ }^{c}$ Lancashire Cardiac Center, Blackpool Victoria Teaching Hospital, Blackpool, United Kingdom; and ${ }^{\mathrm{d}}$ Department of Cardiac Surgery, Hôpitaux Universitaires Henri Mondor, Assistance Publique-Hôpitaux de Paris, Creteil, France.

Disclosures: The authors reported no conflicts of interest.

The Journal policy requires editors and reviewers to disclose conflicts of interest and to decline handling or reviewing manuscripts for which they may have a conflict of interest. The editors and reviewers of this article have no conflicts of interest.

Drs Couetil and Nappi contributed equally to this article.

Received for publication July 6, 2021; accepted for publication Sept 14, 2021; available ahead of print Sept 17 , 2021.

Address for reprints: Francesco Nappi, MD, Cardiac Surgery, Centre Cardiologique du Nord de Saint-Denis, 36 Rue des Moulins Gémeaux, 93200 Saint-Denis, France (E-mail: francesconappi2@gmail.com).

JTCVS Techniques 2021;10:282-8

2666-2507

Copyright (C) 2021 The Author(s). Published by Elsevier Inc. on behalf of The American Association for Thoracic Surgery. This is an open access article under the CC BY-NC-ND license (http://creativecommons.org/licenses/bync-nd/4.0/).

https://doi.org/10.1016/j.xjtc.2021.09.027
}

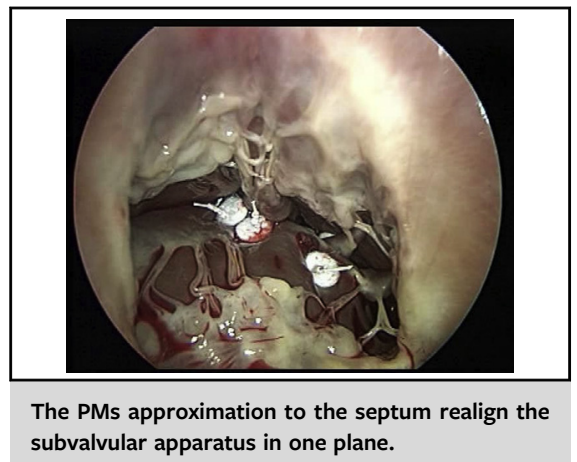

CENTRAL MESSAGE

Tricuspid papillary muscle approximation to the septum combined to annuloplasty reverse the physopathological FTR mechanism.

See Commentaries on pages 289 and 291.
Video clip is available online.

Tenting volume and septal leaflet tethering angle can be considered predictors of severe recurrent tricuspid valve regurgitation after repair. ${ }^{1}$ A tenting area $>1.0 \mathrm{~cm}^{2}$ is predictive of mild tricuspid regurgitation $(\mathrm{TR})^{2}$ and the tethering of the tricuspid leaflets can alone cause TR, even without significant annular tricuspid dilation. ${ }^{3,4}$ While in the mitral counterpart techniques addressing the subvalvular apparatus are widely used, this approach is not commonly adopted for severe functional tricuspid regurgitation (FTR), despite the fact it would be intuitive and justified from the physiological perspective. Preliminary preclinical experiments in large animals using a right-sided papillary sling to reduce tricuspid valve (TV) tethering and ventricular diameters confirmed this hypothesis and showed encouraging results. ${ }^{5}$

We further elaborated this concept and ideated a technique to approximate the papillary muscles (PMs) to the interventricular septum, the so-called septalization of the papillary muscles (PMs-S) of the TV. The rationale of this procedure relies in the possibility to provide a mechanism of correction of FTR addressing the displacement of the PMs and the right ventricular (RV) remodeling process (Figure 1). The selection criteria for performing the PMs-S are reported in Figure E1.

In the majority of cases, the anterior PM is more developed and originates the chordae tendinae destined to the free edge of the anterior and posterior leaflets. Septalization of the anterior papillary muscle is achieved using two CV-4 Gore-Tex sutures reinforced with pledgets passed through the base of the anterior papillary muscle in proximity of its insertion on the free wall during diastolic arrest. By anchoring the anterior papillary muscle to the septum, correction of the TV anterior and posterior leaflet tenting can be achieved (Figure 2). Septalization of the posterior PM can conversely correct septal leaflet tenting (Figure 2 and Video 1). Stabilization of the repair with tricuspid annuloplasty is then achieved routinely.

We present our preliminary proof-of-concept experience with this technique in 11 patients. The institutional review board (CCN_2016-1) approved the study protocol and 

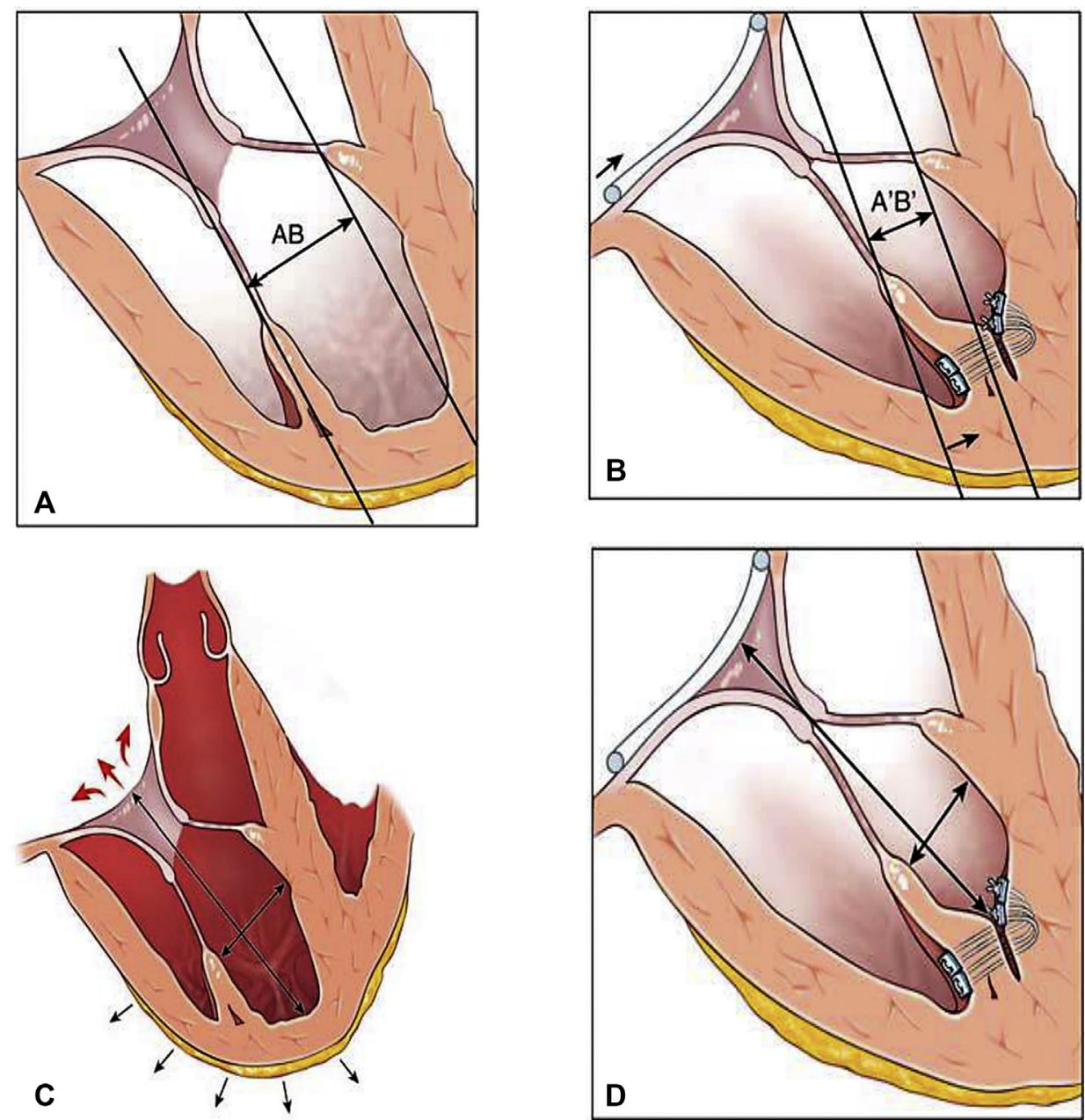

FIGURE 1. A-D, The rationale of the PM septalization. A and B, APM-S treat the tenting by reducing the diameter AB because it allows to bring the tip of the APM closer to the annular plane and to the septum. C and D, APM-S promotes remodeling of the RV by reducing longitudinal and transverse diameters.

publication of data. The patient(s) provided informed written consent for the publication of the study data.

\section{SURGICAL RESULTS}

From 2016 to 2018,11 patients received a papillary muscle septalization for severe FTR $(66.8 \pm 12.8$ years; 6 female). Comprehensive preoperative echocardiographic measurements are shown in Table 1. In summary, preoperative ejection fraction ranged between a minimum of $32 \%$ and a maximum of $68 \%$, end-diastolic left ventricular diameter was a minimum of $32 \mathrm{~mm}$ to a maximum of $68 \mathrm{~mm}$, tricuspid annulus diameter was a minimum of $37 \mathrm{~mm}$ to a maximum of $56 \mathrm{~mm}$, tricuspid tenting height was a minimum of $5 \mathrm{~mm}$ to a maximum of $16 \mathrm{~mm}$, tricuspid tenting area was a minimum of $1.2 \mathrm{~cm}^{2}$ to a maximum of $4.5 \mathrm{~cm}^{2}$, end-diastolic RV diameter was a minimum of $39 \mathrm{~mm}$ to a maximum of $70 \mathrm{~mm}$, pulmonary artery systolic pressure was a minimum of $35 \mathrm{~mm} \mathrm{Hg}$ to a maximum of $58 \mathrm{~mm} \mathrm{Hg}$, and tricuspid annular plane systolic excursion was a minimum of $14 \mathrm{~mm}$ to a maximum of $32 \mathrm{~mm}$. The PM septalization was intraoperatively effective in all patients, as evidenced by the complete leaflets tenting correction at postoperative imaging (Figure 3). Repeat surgery was required for 2 patients (case 3 and 4, Table 1) and 2 patients died at 30 days.

During the 12 months of follow-up, no deaths were recorded. Mild-to-moderate residual TR was observed at the transthoracic echocardiogram control in 1 patient (Table 1, case 9). The other 8 patients experienced a marked reduction in RV size (preoperative minimum of $39 \mathrm{~mm}$ to a maximum $70 \mathrm{~mm}$ vs postoperative minimum of $33 \mathrm{~mm}$ to a maximum of $58 \mathrm{~mm}$ ), pulmonary artery systolic pressure estimated by transthoracic echocardiogram (preoperative minimum of $35 \mathrm{~mm} \mathrm{Hg}$ to a 

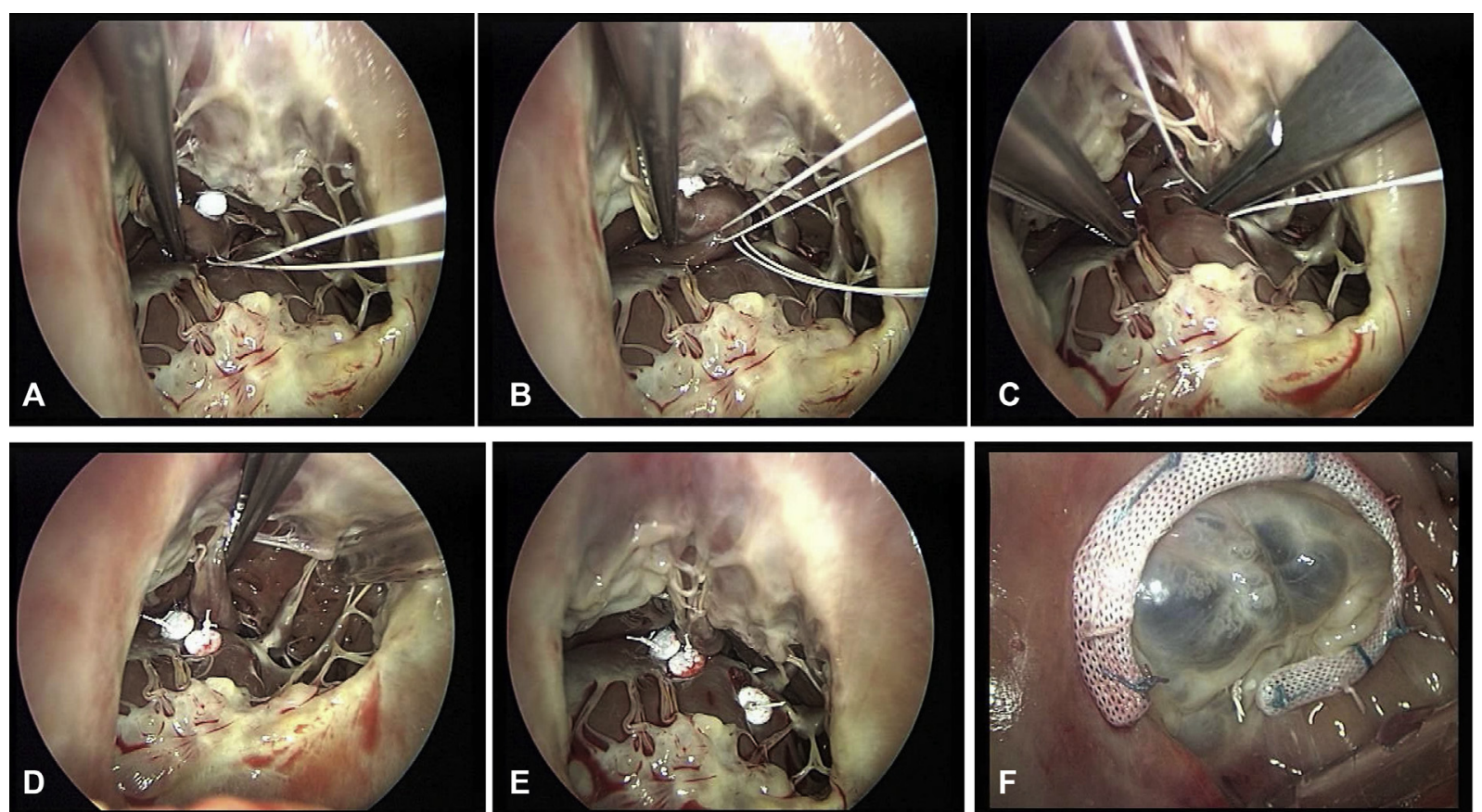

FIGURE 2. A-F, Combined PMs approximation to the septum with TVA. A and B, APM-S using 2 Gore-Tex 4/0 points reinforced with pledget are passed through the base of the PM near its free insertion into the wall. C and D, The APM is brought to the septum and the sutures deeply anchored to the septum, firmly attached, and tied. E, PPM-S is required to treat the tenting of septal leaflet due to PPM displacement. F, PMs-S result shows good coaptation and no more leak.

maximum of $58 \mathrm{~mm} \mathrm{Hg}$ vs a minimum of $23 \mathrm{~mm} \mathrm{Hg}$ to a maximum of $50 \mathrm{~mm} \mathrm{Hg}$ ), and New York Heart Association class.

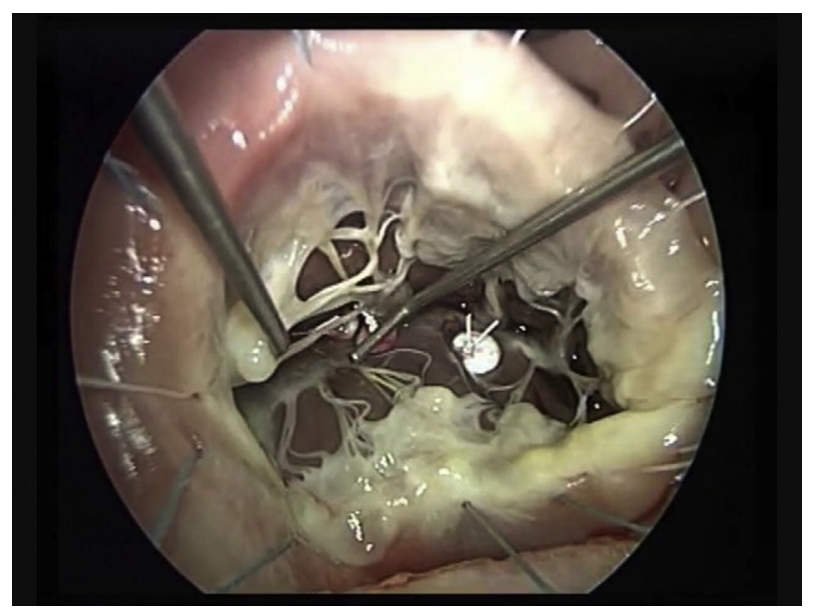

VIDEO 1. The pathophysiology of tricuspid regurgitation implies annular dilation, right ventricular dilatation with papillary muscle dislocation, and consequent leaflet tethering. Tricuspid septalization provides a mean to correct these geometrical abnormalities via approximating the papillary muscle to the interventricular septum. The intraoperative sequence entails the use of Gore-Tex CV-4 sutures reinforced with pledgets passed through the base of the anterior papillary muscle in proximity of its insertion on the free wall which are anchored into the septum. A routine annuloplasty to stabilize the repair is then performed. Video available at: https://www. jtcvs.org/article/S2666-2507(21)00657-X/fulltext.

\section{DISCUSSION}

The mechanism underlying recurrent $\mathrm{TR}$ after annuloplasty is related to the persistent leaflet tethering, as occurs after mitral annuloplasty. Papillary muscle displacement was reported among the factors, reducing the durability of tricuspid annuloplasty in $12 \%$ to $30 \%$ of the cases. ${ }^{1,2}$ Fukuda and colleagues ${ }^{1,2}$ showed that tenting height greater than $5.1 \mathrm{~mm}$ or tenting area greater than $0.8 \mathrm{~cm}^{2}$ predicts moderate recurrent TR after annuloplasty. In our series, preoperative mean tenting height preoperatively was $8.3 \pm 4.1 \mathrm{~mm}$ and tenting area $3.1 \pm 1 \mathrm{~cm}^{2}$ (Table 1).

The use of the papillary muscle approximation procedure has been shown to be safe and effective both for the improvement of the dislocation of the papillary muscles and for the recovery of left ventricular geometric distortion in ischemic mitral regurgitation. ${ }^{6,7}$

Unlike the papillary muscle approximation in which the PMs of the mitral valve are closely connected to the left ventricle in a high-pressure regime, in the PMs-S of the tricuspid valve they are connected to the free ventricular wall by loosened trabeculation in a low-pressure ventricle. The base of the PMs is firmly anchored to the septum and does not hinder the infundibular outflow tract of the right ventricle, thus reducing the risk of potential RV outflow tract obstruction.

The results of tricuspid valve annuloplasty (TVA) are burdened by a significant rate of TR recurrence over 
TABLE 1. Clinical summaries

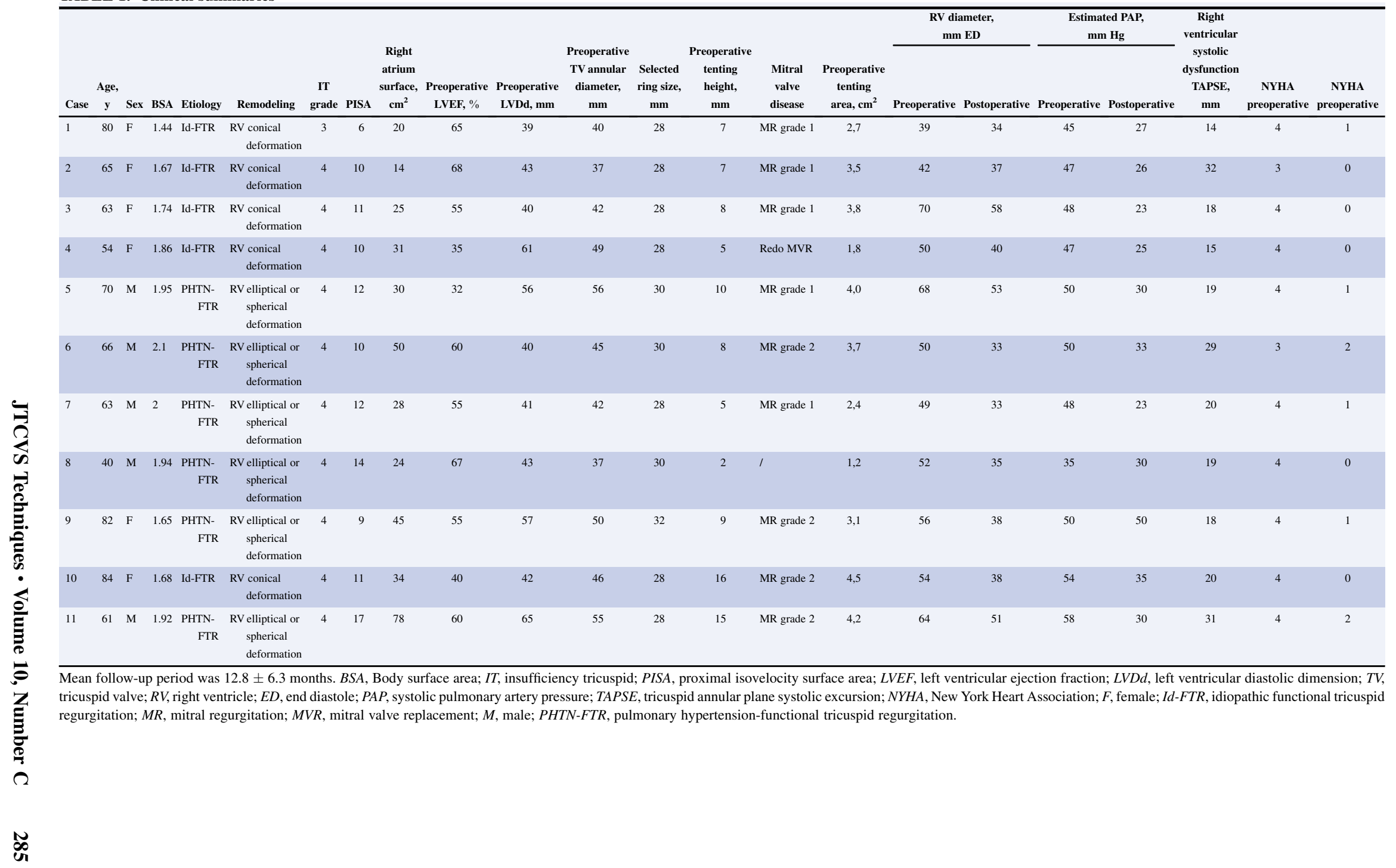



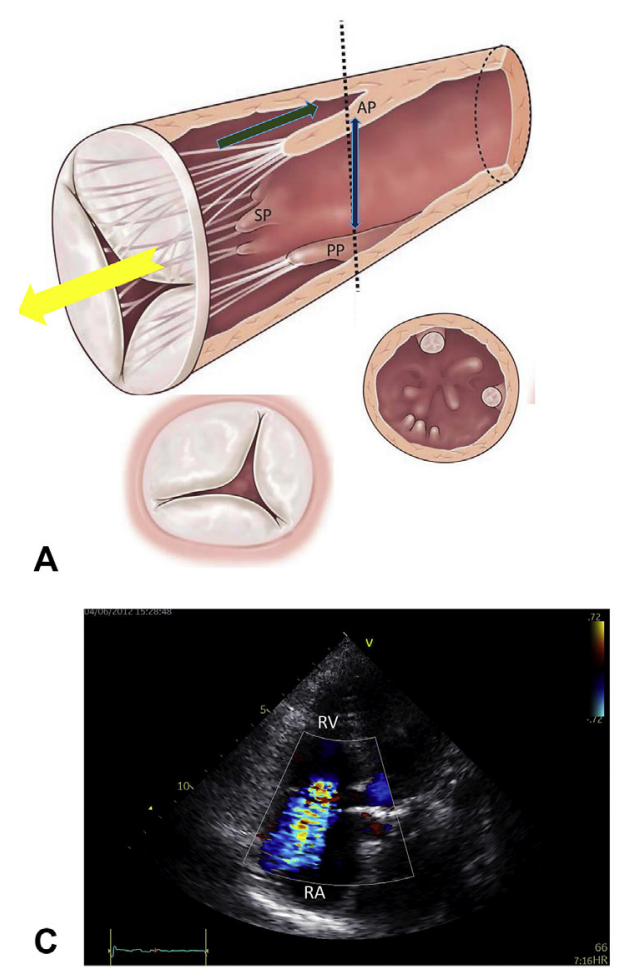
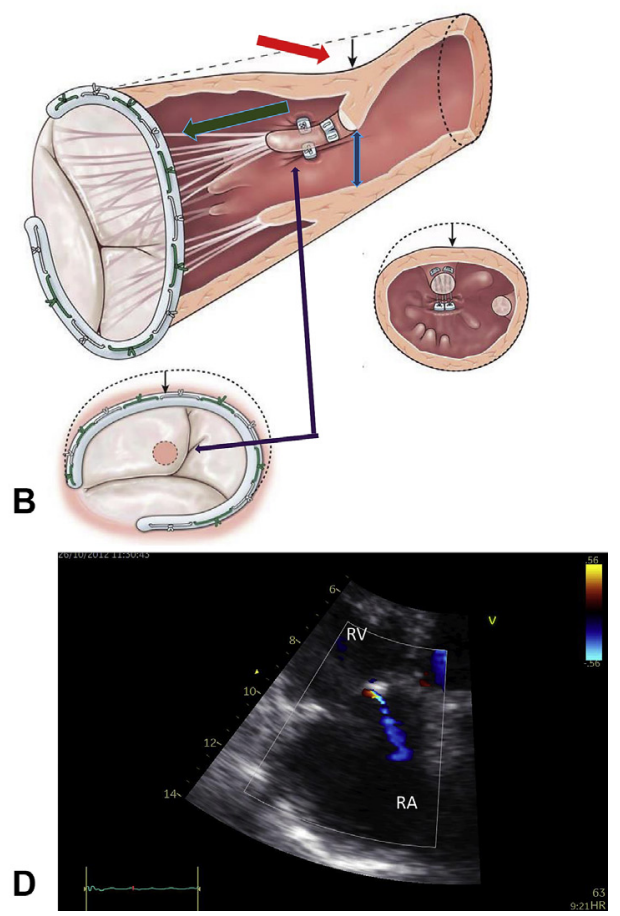

FIGURE 3. Summary of the effect of septalization plus annuloplasty in reversing the mechanism of severe FTR (Table 1, case 1). A, Anatomical changes related to TVR (yellow arrow) involve tricuspid annulus, leaflet, and subvalvular apparatus. Annular dilatation is associated to leaflet tethering (green arrow) due to PMs displacement (blue arrow). B, TVA + APM-S (approximation of APM to the interventricular septum) corrects leaflet tethering (green arrow), displacement of PMs (blue arrow), and RV remodeling (red arrow), eliminating leaflet tenting (purple arrow). C, Preoperative color flow transthoracic echocardiogram, apical 4-chamber view showing significant tricuspid regurgitation with a large central jet. D, Postoperative transthoracic echocardiogram showing a trivial tricuspid regurgitation. $R V$, Right ventricular; $R A$, right atrium.

time, as reported in many series. ${ }^{8-10}$ The correction of leaflet tenting and displacement of PMs due to the progressive right ventricle negative remodeling can be key in preventing a recurrence of TR. In fact, TVA cannot address the distortion of RV geometry and migration of the PMs occurring at the subvalvular level during remodeling. Over time, this phenomenon is considered among the main culprit of recurrence of TR despite the restrictive annular action exerted by the annuloplasty ring.

The PMs of tricuspid valve can be easily mobilized, and we believe that anchoring PMs to the septum could restore right ventricle geometry, as shown by the measurement of $\mathrm{RV}$ diameter, improve remodeling of the right anterior ventricular wall, and decrease RV sphericity with evident improvement in dynamics.

PMs-S is a relatively easy and reproducible procedure because the PMs are fixed on the septum where they "naturally" sit in the empty right ventricle. However, this technique might be more challenging in the instance of bifid or trifid morphology of the posterior papillary muscle. In these cases, a muscle sling would be recommendable. It is important to note that all the patients here reported were operated on crossclamp and no other factor precluded the use of the septalization procedure.

In conclusion, septalization of tricuspid PMs combined with tricuspid valve annuloplasty is a reliable and reproducible technique with no particular contraindications. It allows to correct the leaflet tenting, favors right ventricle remodeling, and decreases the incidence of early and recurrent postoperative FTR. The procedure can find indication particularly in patients with pulmonary arterial pressure reaching $50 \mathrm{~mm} \mathrm{Hg}$ or greater. In these hemodynamic circumstances, the isolated TVA can be burdened by a high recurrence of tricuspid valve regurgitation. Further studies are required to confirm these preliminary proof-of-concept results and improvements in imaging techniques to evaluate the RV remodeling are warranted.

\section{References}

1. Fukuda S, Song JM, Gillinov AM, McCarthy PM, Daimon M, Kongsaerepong V, et al. Tricuspid valve tethering predicts residual tricuspid regurgitation after tricuspid annuloplasty. Circulation. 2005;111:975-9.

2. Fukuda S, Gillinov AM, Song JM, Daimon M, Kongsaerepong V, Thomas JD, et al. Echocardiographic insights into atrial and ventricular mechanisms of functional tricuspid regurgitation. Am Heart J. 2006;152: 1208-14. 
3. Spinner EM, Shannon P, Buice D, Jimenez JH, Veledar E, Del Nido PJ, et al. In vitro characterization of the mechanisms responsible for functional tricuspid regurgitation. Circulation. 2011;124:920-9.

4. Topilsky Y, Khanna A, Le Tourneau T, Park S, Michelena H, Suri R, et al. Clinical context and mechanism of functional tricuspid regurgitation in patients with and without pulmonary hypertension. Circ Cardiovasc Imaging. 2012;5: 314-23.

5. Al-Attar N, Hvass U. Right papillary muscle sling: proof of concept and pilot clinical experience. Eur J Cardiothorac Surg. 2013;43:e187-9.

6. Nappi F, Lusini M, Spadaccio C, Nenna A, Covino E, Acar C, et al. Papillary muscle approximation versus restrictive annuloplasty alone for severe ischemic mitral regurgitation. J Am Coll Cardiol. 2016;67:2334-46.

7. Nappi F, Spadaccio C, Nenna A, Lusini M, Fraldi M, Acar C, et al. Is subvalvular repair worthwhile in severe ischemic mitral regurgitation? Subanalysis of the
Papillary Muscle Approximation trial. J Thorac Cardiovasc Surg. 2017;153: 286-95.e2.

8. Algarni KD, Alfonso J, Pragliola C, Kheirallah H, Adam A, Arafat AA. Longterm outcomes of tricuspid valve repair; the influence of the annuloplasty prosthesis. Ann Thorac Surg. November 23, 2020 [Epub ahead of print].

9. Xu J, Han J, Zhang H, Meng F, Luo T, Tian B, et al. Risk factors for postoperative recurrent tricuspid regurgitation after concomitant tricuspid annuloplasty during left heart surgery and the association between tricuspid annular circumference and secondary tricuspid regurgitation. BMC Cardiovasc Disord. 2021;21:50

10. Shinn SH, Dayan V, Schaff HV, Dearani JA, Joyce LD, Lahr B, et al. Outcomes of ring versus suture annuloplasty for tricuspid valve repair in patients undergoing mitral valve surgery. J Thorac Cardiovasc Surg. 2016;152: 406-15.e3. 


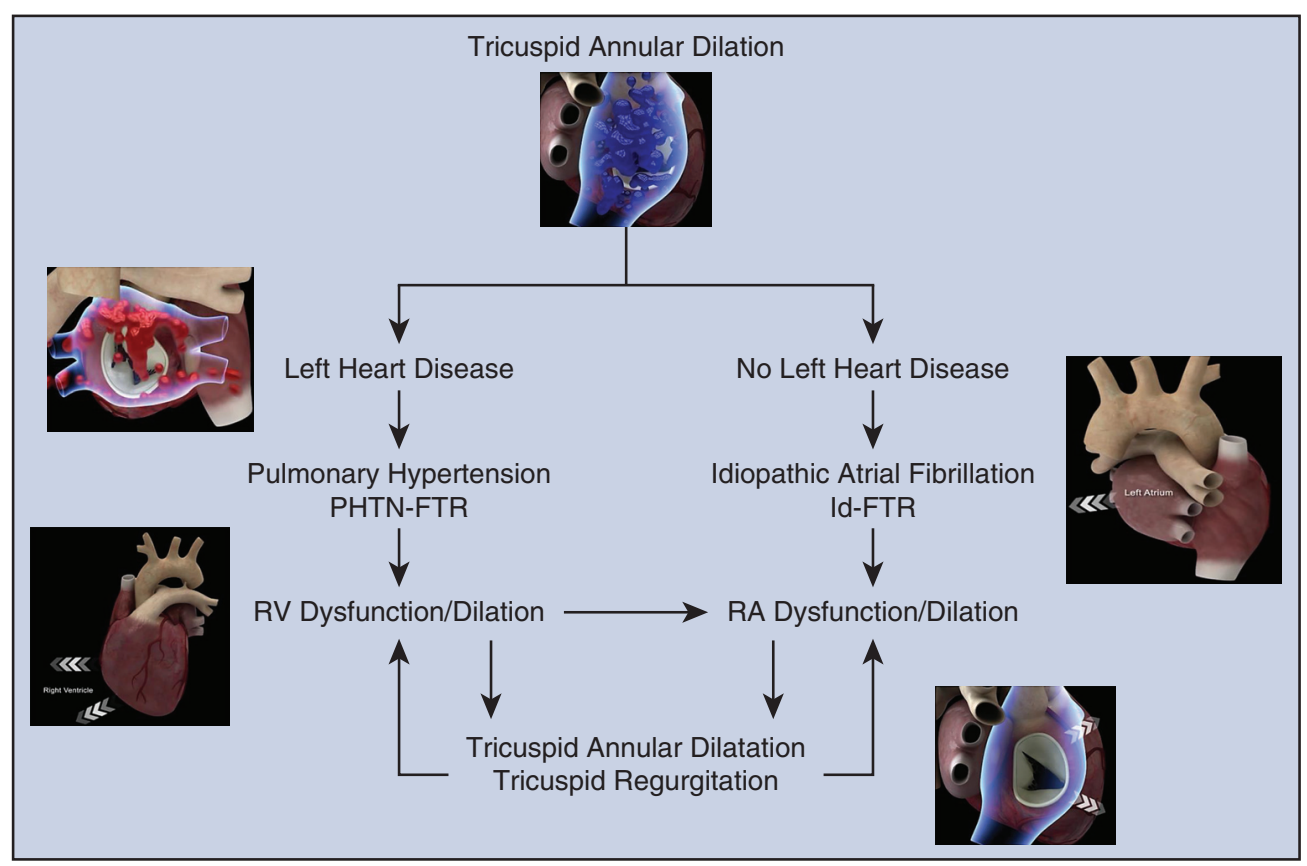

FIGURE E1. Selection criteria to the use of combined PMs-S and TVA in patients with severe TVR. The main selection criterion is severe dilation of the tricuspid annulus with or without LHD. The benefit of the combined approach using PMs-S and TVA is suggested in patients with or without LHD. Patients with pulmonary hypertension and patients with idiopathic atrial fibrillation can greatly benefit from the procedure with improvement of TV dilation and TVR. PHTN-FTR, Pulmonary hypertension-functional tricuspid regurgitation; Id-FTR, idiopathic functional tricuspid regurgitation; $R V$, right ventricle; $R A$, right atrium. 